\title{
SARS-CoV-2 may persist in digestive tract longer than respiratory tract
}

Yilin HU M.D. ${ }^{1 \S}$, Lisha SHEN M.D. ${ }^{2 \S}$, Zheming XU M.D. ${ }^{3}$, Jianying ZHOU M.D. ${ }^{2 *}$, Hua ZHOU M.D. ${ }^{2 *}$

1. Respiratory Department, Deqing People's Hospital;

2. Department of Respiratory and Critical Care Medicine, the First Affiliated Hospital, School of Medicine, Zhejiang University;

3. Internal Medicine Unit, Deqing People's Hospital

${ }^{\S}$ Authors contributed to this study equally.

*Corresponding author: Jianying ZHOU, M.D., zjyhz@zju.edu.cn and Hua ZHOU, M.D., zhouhua1@zju.edu.cn

Ethics Approval: This study has been reviewed and approved by the Research Ethics Committee of the Deqing People's Hospital (ref\# LL2020-01), with a waiver of informed consent. 


\begin{abstract}
Covid-19 has now become a public health concern worldwide. The infection primarily involves the respiratory tract. Hitherto, some Covid-19 pneumonia patients carry the viral nucleic acids, and the active virus was detected in stool specimens. The virus discharged with feces is a potential contagious source. In the present study, three Covid-19 respiratory tract infection patients showed no gastrointestinal symptoms, and two were positive for viral nucleic acids in anal swab specimens remained positive 6 and at least 14 days after virus turned negative in the respiratory tract, respectively (details of the patients were listed in Fig 1). Thus, for Covid-19-infected patients with or without gastrointestinal symptoms, viral nucleic acids in stool specimens or anal swab specimens should be focused on for testing in order to decide the isolation duration of the patient.
\end{abstract}




\section{Introduction}

An outbreak of Covid-19, caused by novel coronavirus SARS-CoV-2, started from December 2019 at Wuhan, China ${ }^{1}$, and has spread to all the provinces and 26 countries. SARS-CoV-2, derived from bats, was transmitted to humans through an intermediate host, which is yet undefined. The pattern of human-to-human transmission mainly involved droplet, aerosol, and contact, but not excluding the possibility of fecal-oral transmission. In the first reported Covid-19 case of the USA, Michelle et al. detected viral nucleic acid in the stool specimen ${ }^{2}$. This indicated the possibility of transmission of virus discharged from the bowel. However, during hospital isolation of this patient, viral nucleic acid in the stool specimen rapidly turned negative as compared to that in the respiratory samples, indicating a low possibility of community contamination.

From January 24-26, 2020, we received three mild cases of Covid-19-infected patients with travel history to Wuhan. After nucleic acid testing turned negative in the respiratory specimens, anal swabs of 2 cases remained persistently positive for 6 and 12 days, respectively, thereby indicating that a subset of patients might still discharge virus through the digestive tract after its elimination from the respiratory tract, rendering it as a source of transmission.

\section{Case}

Case 1, a 28-year-old female, checked in on January 24, 2020 with $>6$ h of fever, accompanied by rhinorrhea, without shortness of breath, chest pain, fatigue, nausea, vomiting, or diarrhea. While breathing ambient air, the patient had finger pulse oxygen saturation of $99 \%$, and no rale was noted in both lungs on auscultation. The maximal body temperature was $38.3{ }^{\circ} \mathrm{C}$. Laboratory tests showed WBC $4.1 \times 10^{9} / \mathrm{L}$, neutrophils $3.11 \times 10^{9} / \mathrm{L}$, lymphocytes $0.70 \times 10^{9} / \mathrm{L}$, and hypersensitive C-reactive protein (hs-CRP) $0.5 \mathrm{mg} / \mathrm{L}$. Lung CT displayed small patchy clouding opacity in the 
right lower lobe. The nasopharyngeal swab specimens were tested positive for SARS-CoV-2 nucleic acids by real-time RT-PCR (rRT-PCR). Based on the epidemic history and clinical symptoms, Covid-19 pneumonia was considered, and the patient was isolated at the hospital. Oseltamivir was administered on day 1 for 5 days consecutively. On day 7 of the illness, the body temperature resumed to normal, and rhinorrhea was improved. On illness day 15 , nasopharyngeal swab specimens turned negative for viral nucleic acids and remained negative when tested sequentially three times over an interval of $>24 \mathrm{~h}$. On the same day, anal swab specimens were collected for the first time for rRT-PCR. These tested positive for SARS-CoV-2 nucleic acids sequentially three more times at an interval of $>24 \mathrm{~h}$. On illness day 26 , anal swab specimens were negative for viral nucleic acids. Strikingly, negative Covid-19 turned was an obvious delay in anal swabs than in nasopharyngeal swabs. The patient will be discharged from isolation after confirming that the nucleic acids were negative in both anal and nasopharyngeal swabs.

Case 2, a 25-year-old male, checked in on January 24, 2020, for 1 day of itchy throat and cough and 6 hours of fever, albeit without shortness of breath, chest pain, fatigue, nausea, vomiting, or diarrhea. When breathing ambient air, the patient had finger pulse oxygen saturation of $94 \%$, and no dry or wet rale was noted in both lungs on auscultation. The maximal body temperature was $38.5^{\circ} \mathrm{C}$. The laboratory tests showed WBC $8.3 \times 10^{9} / \mathrm{L}$, lymphocytes $0.53 \times 10^{9} / \mathrm{L}$, and hs-CRP $0.9 \mathrm{mg} / \mathrm{L}$. Lung CT did not display any lesion. Next, nasopharyngeal swab specimens were tested positive for SARS-CoV-2 nucleic acids by rRT-PCR. Given the epidemic history and clinical symptoms, Covid-19 infection was considered, and hence, the patient was isolated at the hospital. Oseltamivir was used on the first day for 5 days. Cough and fever lasted 6 days. On illness day 11, nasopharyngeal swab specimens turned negative for viral nucleic acids, and similar results were obtained for three consecutive tests conducted at an interval of $>24 \mathrm{~h}$. On illness day 12 , anal swab specimens tested positive for Covid-19 nucleic acids two consecutive times, with the interval of $>24 \mathrm{~h}$. On illness day 16, anal swab specimens turned negative for Covid-19 nucleic acids and continued for two sequential tests. The time when Covid-19 nucleic acids turned 
negative was a 5-day delay in anal swabs than in nasopharyngeal swabs. The patient was discharged from isolation only after confirming that the nucleic acids were negative in both anal and nasopharyngeal swabs.

Case 3, a 32-year-old male, checked in on January 26, 2020, for 2 days of mild fever and cough, without shortness of breath, chest pain, fatigue, nausea, or vomiting. When breathing ambient air, the patient had finger pulse oxygen saturation of $96 \%$, and clear breathing sound was noted in both lungs on auscultation. The maximal body temperature was $37.6{ }^{\circ} \mathrm{C}$. Laboratory tests showed WBC $7.1 \times 10^{9} / \mathrm{L}$, neutrophils $5.29 \times 10^{9} / \mathrm{L}$, lymphocytes $0.99 \times 10^{9} / \mathrm{L}$, and hs-CRP $1.8 \mathrm{mg} / \mathrm{L}$. Lung CT displayed no lesion. Nasopharyngeal swab specimens tested positive for SARS-CoV-2 nucleic acids by rRT-PCR. Owing to the epidemic history and clinical symptoms, Covid-19 infection was considered, and the patient was isolated at the hospital. Oseltamivir was used on the first day for 5 days. Cough lasted 7 days. On illness day 15, nasopharyngeal swab specimens turned negative for viral nucleic acids in three sequentially tests at an interval of $>24 \mathrm{~h}$. On illness day 12 , anal swab specimens were positive for SARS-CoV-2 nucleic acids. On illness day 15, anal swab specimens turned negative for Covid-19 nucleic acids and remained the same for two sequential tests. Furthermore, the virus nucleic acids turned negative in anal and nasopharyngeal swabs on the same day, and the patient was discharged from isolation.

\section{Discussion}

The transmission of Covid-19 virus occurred via droplet, contact, and aerosol. Infected patients discharged SARS-CoV-2 through the respiratory tract, which became a transmission source. Typically, the patients could be discharged from isolation after the virus tested negative in the respiratory tract. Nonetheless, patients with respiratory infection also discharged the virus through stool specimens. To date, several laboratories have isolated SARS-CoV-2 from patients' stool independently in China $^{3}$. The identification of the virus in stool suggested that contact transmission could be prevented by blocking fecal-oral transmission. During SARS-CoV outbreak, the virus is known to enter the sewer system with feces and form aerosols, which then 
spreads within the building via pipes ${ }^{4}$. In this study, two mild Covid-19-infected patients showed viral nucleic acids persistently in anal swab specimens, even after nasopharyngeal swab specimens turned negative; the time to turn negative in anal swabs delayed up to $>2$ weeks in Case 1. If this patient had been discharged according to the criteria of virus-negative in respiratory tract specimens, that in the stool would become a significant risk of community spread. Thus, close monitoring of viral nucleic acids in feces or anal swabs of Covid-19-infected patients should be treated as a vital index for treatment and observation. Moreover, the appropriate disposal of their stool specimens is imperative.

\section{Reference:}

1. Zhu N, Zhang D, Wang W, et al. A Novel Coronavirus from Patients with Pneumonia in China, 2019. New England Journal of Medicine 2020;382:727-33.

2. Holshue ML, DeBolt C, Lindquist S, et al. First Case of 2019 Novel Coronavirus in the United States. New England Journal of Medicine 2020.

3. Xu KJ, Cai HL, Shen YH, et al. Management of corona virus disease-19 (COVID-19): the Zhejiang experience. Journal of Zhejiang University (Medical Sciences)[J], 2020, 49(1): 0-0

4. Peiris JS, Yuen KY, Osterhaus AD, Stohr K. The severe acute respiratory syndrome. N Engl J Med 2003;349:2431-41. 


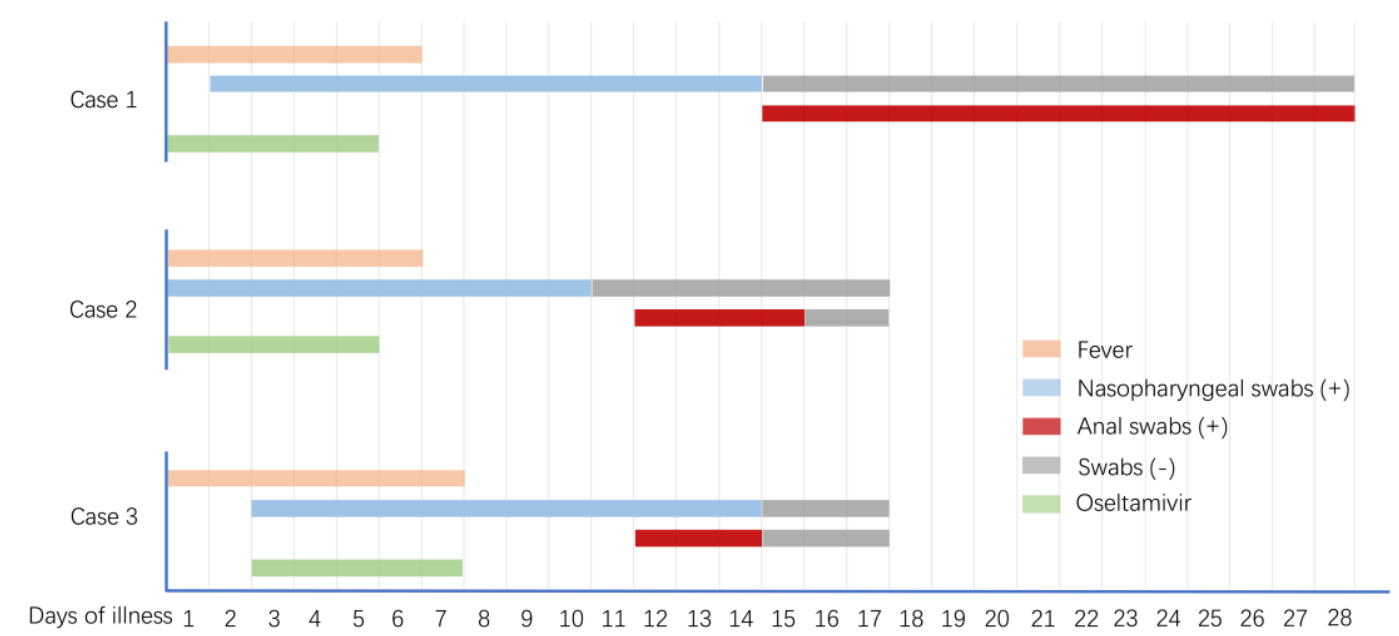

Figure 1. Clinical features alteration of the three cases according to day of illness. The time when SARS-CoV-2 nucleic acids turned negative was $>14$ and 5 days later in the anal swabs than in the nasopharyngeal swabs in cases 1 and 2, respectively. Virus nucleic acids was tested by real-time RT-PCR (rRT-PCR). Oseltamivir was administered in an oral dose of $75 \mathrm{mg}$ twice daily. 